\title{
Microneedles: A Potent Modern Tool in Enhancing Transdermal Drug Delivery
}

\author{
Ogundele $\mathrm{MI}^{1 *}$ and Okafor $\mathrm{HK}^{2}$ \\ ${ }^{1}$ Department of Biomedical Engineering, Binghamton University, USA \\ ${ }^{2}$ Department of Biochemistry, College of Medicine, University of Lagos, Nigeria
}

Submission: September 19, 2017; Published: October 23, 2017

*Corresponding author: Ogundele MI, Department of Biomedical Engineering, Binghamton University, USA, Email: miogundele@gmail.com

\begin{abstract}
The conventional drug delivery methods commonly used are oral administration and injection. However there a limitation posed against the penetration of these substances in therapeutic amounts via the corneum stratum. There has been an increasing emphasis on Transdermal drug delivery which provides a better prospect than the two conventional methods. Transdermal drug delivery provides quite a number of merits which include sustained release, prevention of gastric irritation, improvement in patience compliance as well as removal of pre-systemic first-pass effect. In recent times, microneedles are being proposed for use in enhancing Transdermal drug delivery as the use of microneedles increases skin permeability. This review is a qualitative attempt as discussing the microneedle as a potent modern tool in enhancing transdermal drug delivery. This starts with the explanation of the skin and microneedles. Then the mechanism of action of microneedles, the advantages and disadvantages of microneedles are also discussed. The review ends with the recent advances in the application of microneedles in transdermal drug delivery.
\end{abstract}

\section{Introduction}

When oral administration of drugs is not feasible because of poor drug absorption or enzymatic degradation in the gastrointestinal tract or liver, injection using a painful hypodermic needle is the most common alternative. An approach that is more appealing to patients and offers the possibility of controlled release over time is drug delivery across the skin using a patch [1]. However, transdermal delivery is severely limited by the inability of the large majority of drugs to cross skin at therapeutic rates given the great barrier imposed by skin's outer stratum corneum (SC) layer. Chemical/lipid enhancers [2], electric fields using iontophoresis and electroporation [3], and pressure waves generated by ultrasound or photo acoustic effects [4] are some of different approaches to increase permeability of skin have been studied. Although the mechanisms are all different, these methods share the common goal of disrupting SC structure to create "holes" big enough for molecules to pass through. The size of disruptions generated by each of these methods is believed to be of nanometer dimensions, which are large enough to permit transport of small drugs and, in some cases, macromolecules but probably small enough to prevent damage of clinical significance.

Microneedles are used in clinical practice to deliver medications across the skin into the bloodstream. Injections with hypodermic needles are important from a clinical standpoint, but painful [5]. They may also induce hypersensitivity; bruising, discomfort and bleeding at the site of administration, and in some cases are associated with risks of contamination [5]. There are other concerns linked to their use including accidental needle stick injury and the necessity to train medical staff regarding the proper use of needles [5]. The difficulty in crossing the skin is caused by its anatomical peculiarities. Microneedle arrays markedly increase the skin permeability by up to 3 orders of magnitude [6]. Teo [7] reviewed microneedle designs that are currently being used for transdermal drug delivery. Arrays of microscopic needles create larger transport pathways of micron dimensions. These pathways are orders of magnitude larger than molecular dimensions and, therefore, should readily permit transport of macromolecules and possibly supramolecular complexes and micro particles. Despite their very large size relative to drug dimensions, on a clinical scale these pathways are small. Although safety studies need to be performed, it is proposed that micron-scale holes in the skin are likely to be safe, given that they are smaller than holes made by hypodermic needles or minor skin abrasions encountered in daily life [8].

\section{The skin}

The skin is the largest organ of the body [9], which accounts for more than $10 \%$ of body mass, and the one that enables the body to inter act more intimately with its environment. The skin consists of 4 layers. 


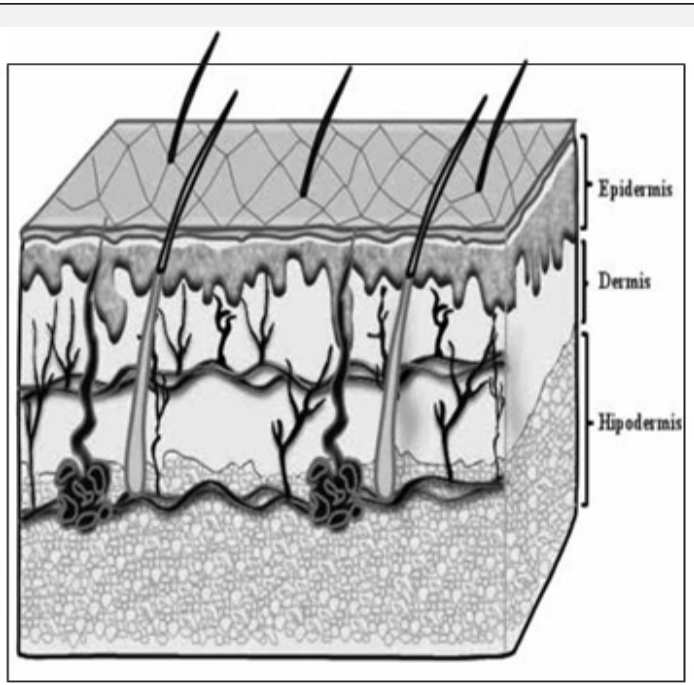

Figure 1: Schematic representation of the skin layers [20].

The SC, the outer layer of the skin (non-viable epidermis), forms the main barrier for diffusion for almost all compounds. It is composed of dead, flattened, keratin rich cells, the corneocytes. These dense cells are surrounded by a complex mixture of intercellular lipids, namely, ceramides, free fattyacids, cholesterol, and cholesterolsulfate. Their most important feature is that they are structured as ordered bilayer arrays [10]. The predominant diffusional path for a molecule crossing the SC appears to be intercellular [11]. The other layers are there maining layers of the epidermis (viable epidermis), the dermis, and the subcutaneous tissues (Figure 1). Associated appendages include hair follicles, sweat ducts, apocrineglands, and nails. Inageneralcon text, the skin's functions may be classified as protecting, maintaining home ostasis, and sensing [12]. A lot of agents are being applied to the epidermal layer of the skin intentionally or accidentally, resulting to either beneficial or harmful effects.

The pertinent focus in dermal absorption assess mentis linked to

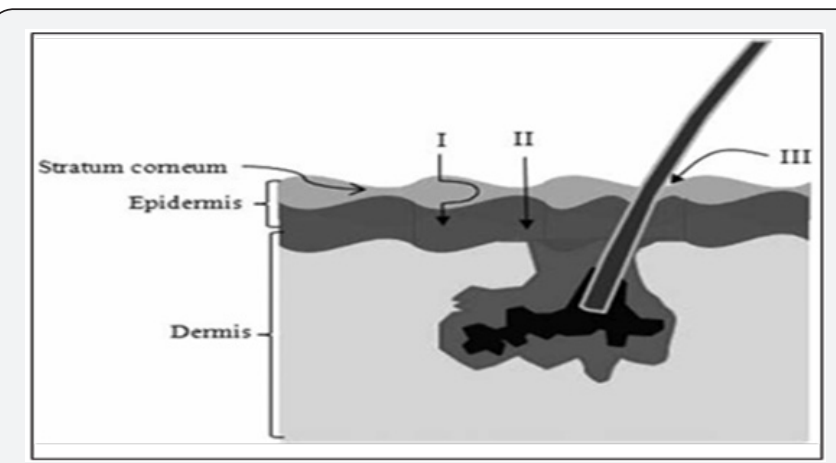

Figure 2: Processes of percutaneous absorption and transdermal delivery (I: Intercellular route, II: Transcellular route and III: Follicular route [20].

A. Passage via the skin to yield asystemic effect (eg: Nicotinepatches, Fentanylpatches)

\section{B. Dermatological local effects (eg:} Corticosteroidsfordermatitis)

C. Surface effects (eg: Sunscreens and cosmetics,)[13]

D. Targeting of deeper tissues (eg: Non-steroidalant iinflammatory agents)[14-18]; and

E. Unwanted absorption (eg: Solvents in the workplace, Pesticides, Orallergens) [19,20]. Figure 2 depicts the pathways of percutaneous absorption.

The skin is popular as a potential site for systemic drug delivery, on the one hand because of the possibility of avoiding the problems of stomach emptying, $\mathrm{pH}$ effects, enzyme deactivation associated with gastro intestinal passage, and hepatic first-pass metabolism and, on the other hand ,because it enables input control.

\section{Microneedles}

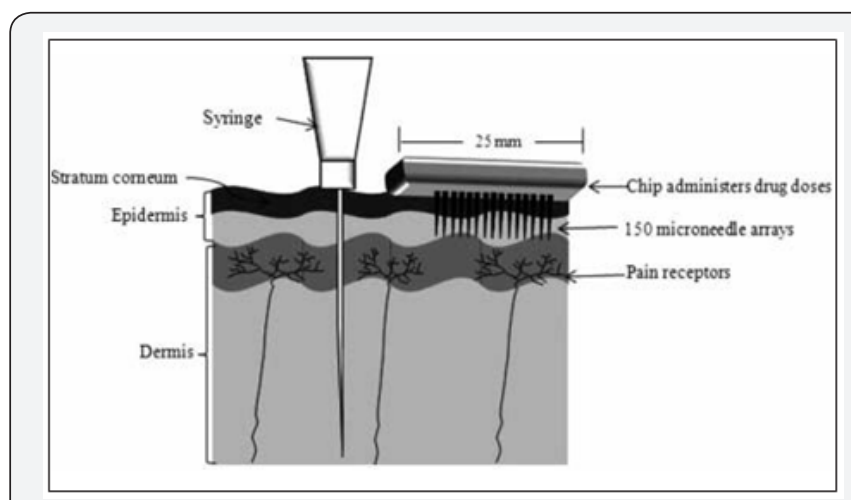

Figure 3: Comparison of a hypodermic syringe and microneedle arrays [20].

Since the past 100 years, hypoderm microneedles as well as syringes have been utilized to deliver drugs to patients. The year 1844 was the first time the hollow needle was invented, and the first injection was delivered shortly after [21], revolutionizing the practice of medicine (Figure 3). The needle's still exerts a strong impact today as a drug delivery vehicle. This is partly because there is a poor absorption of many pharmaceutical in the intestine while some are very sensitive to enzymatic degradation and thus cannot be administered orally.

The syringe and hypodermic needle over the year's have evolved into a 2-part device which is disposable. In clinical practice, the syringe is typically made of plastic and the needle is made of medical-grade stainless steel. The smallest needles available for injections are used largely for insulin administration, measuring 30 gauge for conventional syringes and 31 gauge for pen injectors.

Microneedles defined as micron-scale needles that are used for transdermal vaccination and drug delivery [22]. The possibility that very small needles may be sufficient for transport across the 10 - to $20 \mu \mathrm{m}$-thick SC was first proposed in the $1970 \mathrm{~s}$ [23], but progress was delayed largely because of lack often chniquestofabricate such small structures. The pioneer work 
on the utilization of microneedles for transdermal drug delivery was published in the late 1990s [24].

Established techniques of the micro electronics industry are now being adapted and expanded for microneedle fabrication. Earlier designs of micro-needles used silicon as the fabrication material because of easy adaptability to microelectronic fabrication processes. Current designs emphasize metal and polymeric microneedles. Microneedles used in transdermal delivery can be classified into 2 categories: solid and hollow micro-needles [25]. Solid microneedles have been successfully used to deliver proteins, peptides, oligonucleotides, and nanoparticles in vitro and in vivo [25]. Hollow microneedles have a hollow bore, which offers the possibility of rapid bolus dose drug delivery through flow which is pressure-driven.

\section{Mechanisms of microneedles}

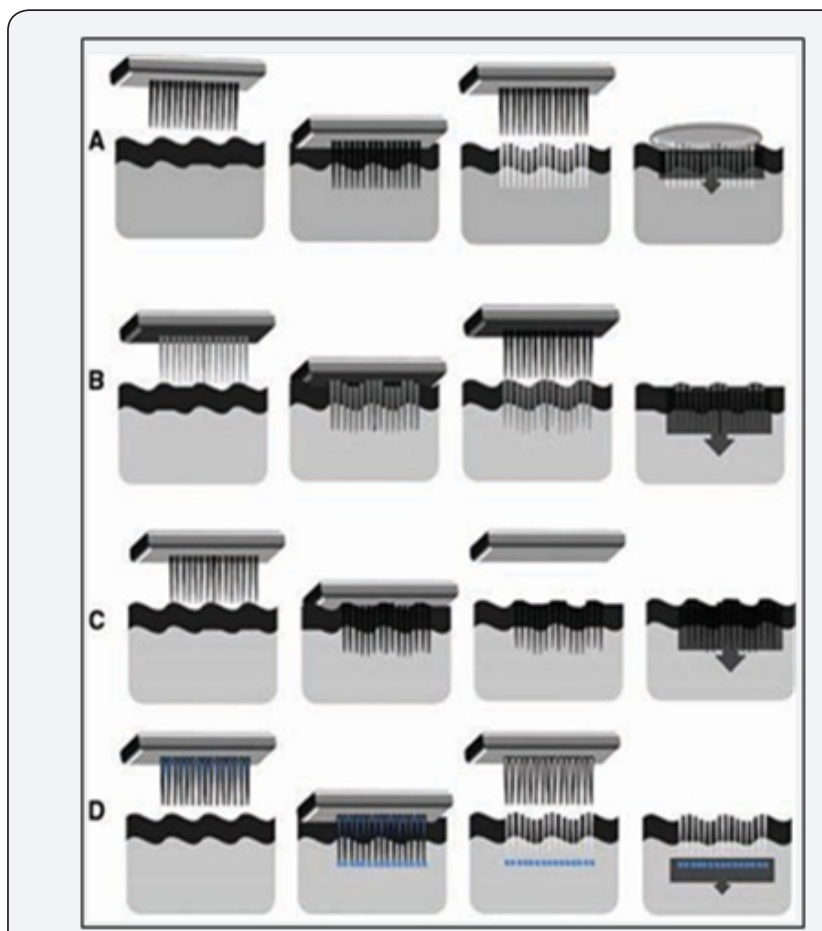

Figure 4: Schematic representation of different microneedle designs for transdermal drug delivery:

A. Solid microneedles,

B. Solid microneedles coated with dry drugs,

C. Polymeric micro- needles with encapsulated drug or vaccine and

D. Hollow micro- needles for injection of drug solution [20].

The mechanism of action depends on the microneedle design and is represented in Figure 4. All the types of microneedles are usually fabricated as an array. Thefinalapproachconsistsofusin ghollowmicro-needlestopuncturetheskinfollowedbyinfusiono fliquidformulationthroughtheneedleboresinamannersimilarto hypodermicinjection [26,27].

\section{Advantages of Microneedles}

The commonest advantage to all physical methods, microneedles inclusive (excluding methods using particle carriers), is that the transport mechanism does not depend on the uptake functions of the cell; therefore, physical methods can be applied equally well to all cell types and at all stages of the cell cycle. The process is, by itself, biologically nontoxic and minimally invasive.

The other advantages of microneedles include the following:

1. Solid microneedles could eventually be used with drug patches to increase diffusion rates; methods to increase permeability include poking holes in skin and rubbing drug over area, or coating needles with drug [28].

2. Microneedles have been fabricated with metals, silicon, silicon dioxide, polymers, glass, and other materials [29]. They can be mass-produced from a range of materials in a consistent and cost-effective manner.

3. Microneedles are fabricated on the micro scale (generally $1 \mu \mathrm{m}$ in diameter, ranging from 1 to $100 \mu \mathrm{m}$ in length) [30].

4. Absence of pain or bleeding makes microneedles more clinically appropriate (particularly in pediatric vaccination and for needle-phobic patients) [29].

5. The mechanism for delivery is not based on diffusion as it is in other transdermal drug delivery products [31]. Placement of the drug or vaccine within the epidermis, where it can more readily reach its site of action.

6. Using microneedles avoids first-pass effect [31]. Microneedles allow rapid penetration of drugs into the systemic circulation.

7. Microneedles can be fabricated to be long enough to penetrate the SC but short enough not to puncture nerve endings [32].

8. Microneedles can provide direct controlled delivery of small molecules, macromolecules, vaccines, or nucleic acids into the viable epidermis [30].

9. Using microneedles reduces the chances of pain, infection, and injury.

10. Hollow needles could eventually be used with drug patches and timed pumps to deliver drugs at specific times.

11. Very small microneedles could provide highly targeted drug administration to individual cells [31].

12. Administration of drugs via microneedles bypasses the gastrointestinal tract.

13. Single-use needles are easily disposable and potentially biodegradable [30].

14. A relatively large surface area can be treated.

15. Drug can be administered at constant rate for a longer period [30]. 


\section{Disadvantages of Microneedles}

In as much as there are lots of advantages of the use of microneedles in transdermal drug delivery however there are a few inherent demerits in its application. They include the following:

1. Microneedles can be difficult to apply on the skin; the clinician must learn proper application technique.

2. Local inflammation may result if the amount of drug is high under the skin. Skin irritation may result because of allergy or sensitive skin [31].

3. The needles are very small and much thinner than the diameter of hair, so the microneedle tips can be broken off and left under the skin [31].

5. Advances in the Applications of Microneedles in Transdermal Drug Delivery

The unique and promising release of drugs by micro-needles in a minimally invasive way renders them an attractive candidate as a physical enhancer to administer drugs throughout the skin $[32,33]$. The following summarizes the research on microneedles used in the transdermal administration of drugs.

\section{$\mu$-0pioid receptor antagonist}

Another hydrophilic and skin impermeable drug that has attracted the attention of researchers is naltrexone (NT), a potent $\mu$-opioid receptor antagonist used to treat opiate and alcohol dependence. Wermeling et al. [34] presented a clinical study for a transdermal patch in healthy human volunteers with and without pretreatment of the skin with microneedles (array of 50 microneedles). Whereas delivery from a standard NT transdermal patch over a 72-hour period yielded undetectable drug plasma levels, pre- treatment of skin with microneedles achieved steady- state plasma concentrations within 2 hours of patch application that were maintained for at least 48 hours. Microneedles and NT patch were well tolerated, with mild systemic and application site side effects. This human proofof-concept study demonstrates systemic administration of a hydrophilic medication by microneedle-enhanced transdermal delivery. These findings set the stage for future human studies of skin-impermeable medications and biopharmaceuticals for clinical applications.

\section{Photosensitizers}

5-aminolevulinic acid (ALA) is used as a protoporphyrinIX precursor for the photo- dynamic therapy of superficial skin cancer and deep or nodular skin tumors. However, the permeability of hydrophilic ALA across the skin is very low.

Donnelly et al. [33] used silicon microneedle arrays (with $6 \times 7$ arrays of microneedles $270 \mu \mathrm{m}$ in height, with a diameter of $240 \mu \mathrm{m}$ at the base and an inter-spacing of $750 \mu \mathrm{m}$ ) to enhance skin penetration of ALA in vitro and in vivo across excised porcine skin. There was a significant increase in transdermal delivery of ALA released from a bioadhesive patch containing 19mg of ALA/ $\mathrm{cm}^{2}$. This clearly has implications for clinical practice, as shorter application times would mean improved patient and clinician convenience and would allow more patients to be treated in the same session. Because ALA is expensive and degrades rapidly via a second order reaction, reducing the required dose is also a notable advantage.

\section{Vasodilators}

The simplest method for reducing the barrier imposed by the SC is to remove it. An adhesive tape removes a layer of corneocytes [35,36]. In vivo, removal of the SC by tape stripping is performed by the repeated application of adhesive tapes to the skin's surface. With this in mind, Sivamani et al. [36] 83 compared topical application of hexyl nicotinate (HN) via injection with hollow microneedle arrays at tape-stripped and unstripped sites in the volar fore- arms of human volunteers. Microneedle injections decreased the time to reach maximum cutaneous blood flow by 3 -fold, regardless of whether the SC had been tape-stripped. Hollow microneedle arrays delivered the agent past the SC and not into the SC. Therefore microneedles improve delivery in animals by penetrating past the SC and would be especially useful in the delivery of lipophilic drugs that partition slowly from the SC into the epidermis.

\section{Dyes (Calcein)}

Oh et al. [37] fabricated biocompatible polycarbonate (PC) microneedle arrays with various depths $(200$ and $500 \mu \mathrm{m})$ and densities $\left(45,99\right.$, and $\left.154 \mathrm{ea} / \mathrm{cm}^{2}\right)$ using a micromechanical process. The skin permeability of a hydrophilic molecule, calcein (622.5D), was examined according to the delivery systems of microneedle and drug loading, with vari-ations in depth and density of the PC microneedle. The skin permeability of calcein was the highest when the calcein gel was applied to the skin with the $500 \mu \mathrm{m}$-depth PC microneedle simultaneously. In addition, the skin permeability of calcein was the highest when $0.1 \mathrm{~g}$ of calcein gel was coupled to the $500 \mu \mathrm{m}$-depth PC microneedle $\left(154 \mathrm{ea} / \mathrm{cm}^{2}\right)$ as well as longer microneedles and larger density microneedles. This study suggests that a biocompatible PC microneedle might be a suitable tool for transdermal drug delivery system of hydrophilic molecules, with possible applications to macromolecules such as proteins and peptides.

Henry et al used a reactive ion-etching micro-fabrication technique to make arrays of microneedles long enough to cross the permeability barrier but not long enough to stimulate nerves. These microneedle arrays could be easily inserted into skin without breaking and increased permeability of human skin in vitro to a model drug, calcein, by up to 4 orders of magnitude. Limited tests on human subjects indicated that microneedles were reported to be painless. This was the first published study on the use of microfabricated microneedles to enhance drug delivery across skin 


\section{Anticancer agents}

Vemulapalli et al. [38] investigated transdermal iontophoretic delivery $\left(0.4 \mathrm{~mA} / \mathrm{cm}^{2}\right.$ applied for 60 minutes) of methotrexate, alone or in combination with maltose microneedle array, in vivo and in vitro by using hairless rat as an animal model. Delivery was enhanced by iontophoresis and microneedles both in vitro and in vivo. A synergistic 25-fold enhancement of delivery was observed in vivo when a combination of microneedles and iontophoresis was used compared with either modality alone.

\section{Calcium channel blockers}

Kolli \& Banga [39] characterized solid maltose microneedles and assessed their ability to increase transdermal drug delivery of nicardipine hydrochloride $(\mathrm{NH})$ in vitro and in vivo across hairless rat skin. Transepidermal water loss was measured to study the skin barrier recovery after treatment. Uniformity in calcein uptake by the pores was characterized, and percutaneous penetration of $\mathrm{NH}$ was studied in vitro and in vivo across hairless rat skin. Microneedles penetrated the skin while creating micro channels measuring about $55.42 \pm 8.66 \mathrm{~mm}$ in diameter. $\mathrm{NH}$ in vitro transport across skin increased significantly after pretreatment (flux $7.05 \mathrm{mg} / \mathrm{cm}^{2} \bullet \mathrm{h}$ ) compared with the untreated skin (flux $1.72 \mathrm{mg} / \mathrm{cm}^{2} \bullet \mathrm{h}$ ) and the enhanced delivery was also demonstrated in vivo in hairless rats.

\section{Insulin}

Microneedles have been widely used in the administration of insulin $[40,41]$. During the past few years, considerable effort has been put into developing novel, more comfortable routes of insulin administration (eg. gastrointestinal, nasal, and inhalation therapy). 89,90 Gastrointestinal and nasal administrations have so far been unsuccessful, whereas inhalation therapy has been successful. However, the inhalation devices are impractical in size, and the long-term safety of inhaled formulations has not been evaluated. Recently, attention has been drawn to the possibility of using a patch-microneedle hybrid to deliver insulin.

Roxhed et al. [40] designed and tested a patch-like system consisting of hollow microneedles and a drug reservoir with an active electrically controlled dispensing mechanism to achieve acceptable delivery rates of insulin in vivo in diabetic rats. Continuous active infusion caused significantly higher insulin concentrations in blood plasma. After a 3-hour delivery period, the insulin concentration was 5 times larger compared with passive delivery. Consistent with insulin concentrations, actively administered insulin resulted in a significant decrease of blood glucose levels. This study shows the feasibility of a patch-like system with on-board liquid storage and dispensing capability. The proposed device is an important step toward painless and convenient administration of macromolecular drugs such as insulin or vaccines.

Nordquist et al. [41] studied a painless intradermal deliver of insulin by using a patch microneedle array in male Sprague
Dawley rats with streptozotocin-induced diabetes. Plasma insulin and blood glucose were measured before, during, and after subcutaneous or intradermal microneedle infusion of insulin $(0.2 \mathrm{IU} / \mathrm{h})$. Administration of insulin resulted in a reduced plasma glucose independent of administration route.

This study presents a novel possibility of insulin delivery that is controllable and requires minimal training. This treatment strategy could improve compliance and thus improve patients' glycemic control. Martanto et al. [42] investigated the design and use of microneedle arrays (105 microneedles from stainless steel) to deliver insulin into the skin of diabetic hairless rats with streptozotocin-induced diabetes. During and after microneedle treatment, an insulin solution $(100$ or $500 \mathrm{U} / \mathrm{mL}$ ) was placed in contact with the skin for 4 hours. Microneedles were removed 10 seconds, 10 minutes, or 4 hours after initiation of transdermal insulin delivery. Arrays of microneedles were fabricated and demonstrated to insert fully into hairless rat skin in vivo. Microneedles increased skin permeability to insulin, which rap-idly and steadily reduced blood glucose levels to an extent similar to 0.05 to $0.5 \mathrm{U}$ of insulin injected subcutaneously. Solid metal microneedles were capable of increasing transdermal insulin delivery and lowering blood glucose levels by as much as $80 \%$ in diabetic hairless rats in vivo.

\section{Heparin}

In some cases, the combination of different active enhancement methods has been effective in increasing the transport of drugs through the skin [43]. For example, Lanke et al. [44] investigated the in vitro transdermal delivery of low molecular weight heparin (LMH) in the hairless rat treated with various enhancement strategies (passive diffusion, iontophoresis $\left[0.5 \mathrm{~mA} \bullet \mathrm{cm}^{2}\right.$ for 4 hours], sonophoresis $[55 \mathrm{kHz}$ for 60 seconds], tape stripping [20 pieces of 3M Transpore adhesive tape removed in each skin section]) and the use of an array of 28 soluble maltose microneedles. Passive flux was essentially zero and remained low even after iontophoresis $\left(0.065 \mathrm{U} / \mathrm{cm}^{2} \bullet \mathrm{h}\right)$ or application of ultrasound $(0.058 \mathrm{U} / \mathrm{cm} 2 \bullet \mathrm{h})$. A significant increase in flux across tape-stripped skin (4.0U/ $\mathrm{cm}^{2} \bullet \mathrm{h}$ ) suggests the interaction of Stratum Corneum. Maltose microneedles were then used to locally disrupt and bypass the Stratum Corneum. Microneedles breached the Stratum Corneum and enhanced LMH permeability $\left(0.175 \mathrm{U} / \mathrm{cm}^{2} \bullet \mathrm{h}\right)$. Microneedles when used in conjunction with iontophoresis had a synergistic effect on LMH delivery, enhancing flux by 14.7 fold compared with iontophoresis used alone. LMWH was shown to interact with Stratum Corneum, and therefore tape stripping or microneedles dramatically increased its delivery by disrupting the SC skin barrier.

\section{Gene therapy and vaccination}

Stratum Corneum (SC) presents a significant barrier to the delivery of gene therapy formulations. To realize the potential of therapeutic cutaneous gene transfer, delivery 
strategies are required to overcome this exclusion effect. For this reason, microneedles have been used for the delivery of membrane-impermeable molecules into cells, for application in molecular cell biology, and for the delivery of peptides, proteins, oligonucleotides, DNA, and other probes that alter or assay cell function.

Coulman et al. [45] studied the ability of microfabricated silicon microneedle arrays to create micron- sized channels through the Stratum Corneum of ex vivo human skin and the resulting ability of the conduits to facilitate localized delivery of charged macromolecules and plasmid DNA (pDNA). The delivery of a macromolecule, $\beta$-galactosidase, and of a "non-viral gene vector-mimicking" charged fluorescent nano-particle to the viable epidermis of microneedle-treatedtissue was demonstrated using light and fluorescent microscopy. Permeation profiles showed that more than $50 \%$ of a colloidal particle suspension permeated membrane pores in approximately 2 hours. On the basis of these results, it is probable that micro- needle treatment of the skin surface would facilitate the cutaneous delivery of lipid polycation pDNA (LPD) gene vectors, and other related vectors, to the viable epidermis. Gene expression studies confirmed that naked pDNA can be expressed in excised human skin following microneedle disruption of the SC barrier. The presence of a limited number of microchannels positive for gene expression indicates that further studies to optimize the microneedle device morphology, its method of application, and the pDNA formulation are warranted to facilitate more reproducible cutaneous gene delivery.

Li et al. [46] investigated the microneedle-mediated in vitro transdermal delivery of human IgG in full- thickness hairless rat skin. In vitro penetration studies were conducted using freshly excised full-thickness hairless rat skin, and various parameters like needle length, number of needles, and effect of donor concentration were examined. Pathway of IgG transport across skin was confirmed by immune histochemical (IHC) studies.

A monoclonal antibody was delivered under optimized conditions. Methylene blue was taken up by micro channels, indicating disruption of the SC, and cryosections showed that microneedles just reached the dermis. Human IgG delivery increased with increases in arrays of microneedles, concentration, and length of microneedles. IHC studies demonstrated that IgG follows microchannels for transport across the skin. Transdermal delivery was also demonstrated for the monoclonal antibody. Maltose microneedles provide a means for the transdermal delivery of macromolecules.

Trehalose ( $\alpha$-D-glucopyranosyl- $\alpha$-D-glucopyranoside) is recognized as a promising, fast-dissolving, solid reservoir capable of stabilizing the native structure of proteins and suitable for loading with a wide variety of bioactive substances. There is a growing interest in developing cost-effective methods for immobilizing solid trehalose on arrays of microneedles to deliver protein-based and DNA-based vaccine to the epidermis.
Shirkhanzadeh [47] used microporous calcium phosphate coatings to provide a biocompatible interface with a large surface area for the effective immobilization of trehalose on microneedles. The mechanical performance of the coatings was assessed by inserting the tips of the coated needles into human skin to an average depth of 100 to $300 \mu \mathrm{m}$ and then removing them for analysis by scanning electron microscopy. Microporous calcium phosphate coatings loaded with trehalose effectively breached the SC and allowed direct access to the epidermis without breaking and without stimulating nerves in deeper tissues.

Chabri et al. [48] determined whether silicon-based microneedles could generate microchannels in human skin of sufficient dimensions to facilitate access of LPD non-viral gene therapy vectors. The diffusion of fluorescent polystyrene nanospheres and LPD complexes through heat-separated human epidermal sheets was determined in vitro using a Franz-type diffusion cell. In vitro cell culture with quantification by flow cytometry was used to determine gene expression in human keratinocytes (HaCaT cells).

The diffusion of 100-nm-diameter fluorescent polystyrene nanospheres, used as a readily quantifiable predictive model for LPD complexes, through epidermal sheets was significantly enhanced following membrane treatment with microneedles. The delivery of LPD complexes either into or through the membrane microchannels was also demonstrated. In both cases considerable interaction between the particles and the epidermal sheet was observed. These studies demonstrate the utility of silicon microneedles in cutaneous gene delivery.

Combination vaccines reduce the total number of injections required for each component administered separately and generally provide the same level of disease protection. Yet physical, chemical, and bio- logical interactions between vaccine components are often detrimental to vaccine safety or efficacy.

As a possible alternative to combination vaccines, Morefield et al. [49] used specially designed microneedles to inject rhesus macaques with 4 separate recombinant protein vaccines for anthrax, botulism, plague, and staphylococcal toxic shock next to each other just below the surface of the skin, thus avoiding potentially incompatible vaccine mixtures. The intradermally administered vaccines retained potent antibody responses and were well tolerated by rhesus macaques. Tracking of the adjuvant showed that the vaccines were transported from the dermis to draining lymph nodes by antigen-presenting cells. Vaccinated primates were completely protected from an otherwise lethal aerosol challenge by Bacillus anthracis spores, botulinum neurotoxin $A$, or staphylococcal enterotoxin $B$. The vaccination method may be scalable to include a greater number of antigens while avoiding the physical and chemical incompatibilities encountered by combining multiple vaccines together in one product. 
Influenza, a contagious disease caused by a pathogenic virus responsible for outbreaks all over the world and thousands of hospitalizations and deaths every year, has attracted the attention of researchers. Because of virus antigenic drift and short-lived immune responses, annual vaccination is required. Koutsonanos et al. [50] investigated a novel method for transdermal delivery (applied in the caudal dorsal skin area of mice) using metal microneedle arrays coated with inactivated influenza virus (A/ Aichi/2/68, $\mathrm{H}_{3} \mathrm{~N}_{2}$ ) to determine whether this route is a simpler and safer approach than the conventional immunization. Microneedle vaccination induced a broad spectrum of immune responses including CD4+ and CD8+ responses in the spleen and draining lymph node, a high frequency of antigen-secreting cells in the lung, and induction of virus-specific memory B cells. The use of microneedles showed a dose-sparing effect and a strong Th2 bias compared with an intramuscular reference immunization.

Delivery of inactivated influenza virus through the skin using metal microneedle arrays induced strong humoral and cellular immune responses capable of conferring protection against virus challenge as efficiently as intramuscular immunization. In view of the convenience of delivery and the potential for selfadministration, vaccine-coated metal microneedles may provide a novel and highly effective immunization method.

\section{Skin Therapy}

Microneedle skin therapy is still in testing and development, but it seems to show much promise. Microneedle therapy is a way to rejuvenate the skin without destroying the epidermis. It is similar to laser treatments but with less damage. Companies like Clinical Resolution Lab (Los Angeles, California) use treatments involving microrollers [51]. Microneedles penetrate the epidermis and break away old collagen strands. The collagen strands that are destroyed create more collagen under the epidermis. This leads to youthful looking skin. The only disadvantage of this method is that it causes blood oozing, which laser treatments do not. It does, however, have advantages such as increased collagen, non- sun sensitivity upon treatment, no breaking of the epidermis, lower cost, and ease of application.

\section{Conclusion}

This paper has effectively described some of the frontiers in Microneedle as a potent modern tool in enhancement of transdermal drug delivery. This is owing to advances in micro fabrication technology as well as its scalability in a configuration of multi array. Oral treatment has some obvious limitations caused by low surface absorption area and much reduced rate of enzymatic degradation. Hence the advent of microneedle has provided a much more needed solution to these challenges as against oral treatment. Compared with commercially available hypodermic needles, microneedles enable pain-free insertion, minimal tissue damage, and increased control over drug dosage, independent of drug com- position and concentration [1,2].
Buoyed by the numerous advantages of microneedles as already discussed in this literature review, the interest in the use of microneedles is ever increasing presently.

It is also well known that most children have phobia for needles. In addition, many patients suffering from chronic diseases like diabetics receive multiple injections on daily basis while a lot of other disease conditions would require the delivery of therapeutic agents to the skin hence the numerous challenges associated with needle-based injections has been overcome by the development of microneedles [2].

However, valid concerns regarding the use of these microneedles especially safety issues have also been highlighted. There are concerns about costs of the delivery system, delayed onset of action and possible misuse, accidental use, or abuse [52]. There is a need to investigate further skin pore closure after MN application especially as it relates to the risk of infections. It is also essential to ensure that materials which are used for MN fabrication do not induce skin irritation. Another area is the need for a balance between increased permeability and painlessness. It is known that as MN length increases, there is a high probability that pain receptors located in the dermis may be stimulated. In spite of the above-mentioned limitations, the outlook for the use of these devices is promising even as more work needs to be done for microneedles to become routine drug delivery systems in clinical practice.

\section{References}

1. Bronaugh RL, Maibach HI (1999) Percutaneous Absorption: DrugsCosmetics-Mechanisms-Methodology. New York, USA.

2. Cevc G (2004) Lipid vesicles and other colloids as drug carriers on the skin. Adv Drug Deliv Rev 56(5): 675-711.

3. Wang Y, Thakura R, Fan Q Michniak B (2005) Transdermal iontophoresis: combination strategies to improve transdermal iontophoretic drug delivery. Eur J Pharm Biopharm 60(2): 179-191.

4. Mitragotri S, Kost J (2004) Low-frequency sonophoresis: a review. Adv Drug Deliv Rev 56(5): 589-601.

5. Lhernould MS, Deleers M, Delchambre A (2015) Hollow polymer microneedles array resistance and insertion tests. Int J Pharm 480(12): 152-157.

6. Park JH, Allen MG, Prausnitz MR (2005) Biodegradable polymer microneedles: fabrication, mechanics and transdermal drug delivery. J Control Rel 104(1): 51-66.

7. Teo AL (2006) Transdermal microneedles for drug delivery applications. Mater Sci Eng 132(1-2): 151-154.

8. Champion RH, Burton JL, Burns DA, Breathnach SM (1998) Textbook of Dermatology, London, UK.

9. Forslind BA (1994) Domain mosaic model of skin barrier. Acta Derm Venereol 74(1): 1-6.

10. Ellias PM (1991) Epidermal barrier function: intercellular lamellar lipid structures, origin, composition and metabolism. J Control Rel 15(3): 199-208.

11. Guy RH, Hadgraft J (2003) Transdermal Drug Delivery. New York, USA, pp: $1-23$. 
12. Walters KA, Roberts MS (2002) Dermatological and Transdermal Formulations. New York, USA, pp: 1-39.

13. Martínez BIO, Delgadillo JC, Fajardo SBC, García RV, Quintanar AG, et al. (2005) Preparation of polymeric nanocapsules containing octylmethoxycinnamate by the emulsification-diffusion technique: penetration across the stratum corneum. J Pharm Sci 94(7): 15521559.

14. Miyazaki S, Yokouchi C, Nakamura T, Hashiguchi N, Hou WM, et al. (1986) Pluronic F-127 gels as a novel vehicle for rectal administration of indomethacin. Chem Pharm Bull 34(4): 1801-1808.

15. Chi SC, Do K, Tan HK, Chun HW (1996) Anti-inflammatory and analgesic transdermal gel. US Patent No. 5527832.

16. Fang JY, Leu YL, Wang YY, Tsai YH (2002) In vitro topical application and in vivo pharmacodynamic evaluation of nonivamide hydrogels using Wistar rat as an animal model. Eur J Pharm Sci 15(5): 417-423.

17. Shin SC, Cho CW, Oh IJ (2001) Effects of non-ionic surfactants as permeation enhancers towards piroxicam from the poloxamer gel through rat skins. Int J Pharm 222(2): 199-203.

18. Liaw J, Lin YC (2000) Evaluation of poly (ethylene oxide)poly(propylene oxide)-poly(ethylene oxide) (PEO-PPO-PEO) gels as a release vehicle for percutaneous fentanyl. J Control Rel 68(2): 273-282.

19. Chao YCh, Nylander LA (2004) Determination of keratin protein in a tape-stripped skin sample from jet fuel exposed skin. Ann Occup Hyg 48(1): 65-73.

20. Chávez JJE, Martínez BD, González MAV, Trinidad EM, Alancaster NC, et al. (2011) Microneedles: A Valuable Physical Enhancer to Increase Transdermal Drug Delivery. J Clin Pharmacol 51(7): 964-977.

21. Hypodermic injection (1999) The World Book Encyclopedia, Ill: World Book, 9: 480-481.

22. Reed ML, Lye WK (2004) Microsystems for drug and gene delivery. Proc IEEE 92: 56-75.

23. LaVan DA, McGuire T, Langer R (2003) Small-scale systems for in vivo drug delivery. Nat Biotechnol 21(10): 1184-1191.

24. Park JH, Allen MG, Prausnitz MR (2005) Biodegradable polymer microneedles: fabrication, mechanics and transdermal drug delivery. J Control Rel 104(1): 51-66.

25. Prausnitz MR (2004) Microneedles for transdermal drug delivery. Adv Drug Deliv Rev 56(7): 581-587.

26. Gardeniers JGE, Berenschot JW, De Boer MJ, Berenschot EJW, Van Den Berg, et al. (2002) Silicon micromachined hollow microneedles for transdermal liquid transport. IEEE 141-142.

27. Wang PM, Cornwell M, Hill J, Prausnitz MR (2006) Precise microinjection into skin using hollow microneedles. J Invest Dermatol 126(5): 1080-1087.

28. Birchall JC (2006) Microneedle array technology: the time is right but is the science ready? Expert Rev Med Devices 3(1): 1-4.

29. http://www.pharmainfo.net/reviews/microneedles-option-painlessdelivery

30. http: / / ww w.pharmainfo.net/reviews / microneedlesrevolutiontransdermal-drug-delivery

31. http://www.pharmainfo.net/pharma-student-magazine/ happeningstransdermal-drug-delivery-technology

32. Martanto W, Davis SP, Holiday NR, Wang J, Gill HS, et al. (2004) Transdermal delivery of insulin using microneedles in vivo. Pharm Res 21(6): 947-952.

33. Donnelly RF, Morrow DIJ, McCarron PA, Woolfson AD, Morrissey A, et al. (2008) Microneedle mediated intradermal delivery of 5-aminolevulinic acid: Potential for enhanced topical photodynamic therapy. J Control Rel 129(3): 154-162.

34. Wermeling DP, Banks SL, Hudson DA, Harvinder SG, Jyoti G, et al. (2017) Microneedles permit transdermal delivery of a skin-impermeant medication to humans. PNAS 105(6): 2058-2063.

35. Chávez JJE, Contreras LMM, Cervantes LM, Quintanar G, Ganem Q, et al. (2009) The tape stripping technique as a valuable tool for evaluating topical applied compounds. In: Caldwell GW, Atta-ur-Rahman, Yan Z, Choudhary MI (Eds.), Frontiers in Drug Design \& Discovery, The Netherlands, pp. 189-227.

36. Sivamani RK, Stoeber B, Liepmann D, Maibach HI (2009) Microneedle penetration and injection past the stratum corneum in humans. J Dermatolog Treat 20(3): 156-159.

37. Oh JH, Park HH, Do KY, Han M, Hyun DH, et al. (2008) Influence of the delivery systems using a microneedle array on the permeation of a hydrophilic molecule, calcein. Eur J Pharm Biopharm 69(3): 10401045.

38. Vemulapalli V, Yang Y, Friden PM, Banga AK (2008) Synergistic effect of iontophoresis and soluble microneedles for transdermal delivery of methotrexate. J Pharm Pharmacol 60(1): 27-33.

39. Kolli CS, Banga AK (2008) Characterization of solid maltose microneedles and their use for transdermal delivery. Pharm Res 25(1): 104-113.

40. Roxhed N, Samel B, Nordquist L, Griss P, Stemme G (2008) Painless drug delivery through microneedle-based transdermal patches featuring active infusion. IEEE Trans Biomed Eng 55(3): 1063-1071.

41. Nordquist L, Roxhed N, Griss P, Stemme G (2007) Novel microneedle patches for active insulin delivery are efficient in maintaining glycaemic control: an initial comparison with subcutaneous administration. Pharm Research 24(7): 1381-1388.

42. Martanto W, Davis SP, Holiday NR, Wang J, Harvinder SG, et al. (2004) Transdermal delivery of insulin using microneedles in vivo. Pharm Res 21: 947-952.

43. Chávez EJJ, Martínez BD, González A, Vazquez AL (2009) The electroporation as an efficient physical enhancer for transdermal drug delivery. J ClinPharmacol 49(11): 1262-1283.

44. Lanke SSS, Kolli CS, Stroma JG, Banga AK (2009) Enhanced transdermal delivery of low molecular weight heparin by barrier perturbation. Int J Pharm 365(1-2): 26-33.

45. Coulman SA, Barrow D, Anstey A, Gateley C, Morrissey A, et al. (2006) Minimally invasive cutaneous delivery of macromolecules and plasmid DNA via microneedles. Curr Drug Deliv 3(1): 65-75.

46. Li G, Badkar A, Nema S, Kolli CS, Banga AK (2009) In vitro transdermaldelivery of therapeutic antibodies using maltose microneedles. Int J Pharm 368(1-2): 109-115.

47. Shirkhanzadeh M (2005) Microneedles coated with porous calcium phosphate ceramics: effective vehicles for transdermal delivery of solid trehalose. J Mat Sci 16(1): 37-45.

48. Chabri F, Bouris K, Jones T, Barrow D, Hann A, et al. (2004) Microfabricated silicon microneedles for nonviral cutaneous gene delivery. Br J Dermatol 150(5): 869-877.

49. Morefield GL, Tammariello RF, Purcell BK, Worsham PL, Chapman J, et al. (2008) An alternative approach to combination vaccines: intradermal administration of isolated components for control of anthrax, botulism, plague and staphylococcal toxic shock. J Immune Based Ther Vaccines 6: 1-11.

50. Koutsonanos DG, Martin MP, Zarnitsyn VG, Sullivan SP, Compans RW, et al. (2009) Transdermal influenza immunization with vaccine-coated microneedle arrays. PLoS One 4(3): 1-10. 
51. http://www.microneedle.com/main/whatismts.html

52. Salvador CE, Mahmood TM, McElnay JC, McCarthy HO, Mooney K, et al. (2015) Potential of hydrogel-forming and dissolving microneedles for use in paediatric populations. Int J Pharm 489(1-2): 158-169.

Your next submission with Juniper Publishers will reach you the below assets

- Quality Editorial service

- Swift Peer Review

- Reprints availability

- E-prints Service

- Manuscript Podcast for convenient understanding

- Global attainment for your research

- Manuscript accessibility in different formats

( Pdf, E-pub, Full Text, Audio)

- Unceasing customer service

Track the below URL for one-step submission https://juniperpublishers.com/online-submission.php 